\title{
Der Einfluss der sozialen Herkunft auf die internationale Mobilität und den Stellensuchradius von Studierenden und Hochschulabsolventen
}

\author{
Von Fabian Kratz
}

Zusammenfassung: Anhand des mikroökonomischen Humankapitalmodells werden in diesem Beitrag Thesen zur internationalen Mobilität und dem Stellensuchradius von Studierenden und Hochschulabsolventen formuliert. Dabei wird ein besonderer Schwerpunkt auf den Effekt der sozialen Herkunft in Verbindung mit vorherigen Mobilitätserfahrungen gelegt. Mit logistischen Regressionen und Daten des Bayerischen Absolventenpanels durchgeführte Schätzungen zeigen, dass die soziale Herkunft sowohl während des Studiums als auch beim Berufseinstieg einen signifikanten Einfluss auf die Wahrscheinlichkeit internationaler Mobilität von Studierenden und Hochschulabsolventen ausübt. Außer diesem direkten Effekt besteht ein indirekter Effekt darin, dass Studierende und Absolventen mit hoher sozialer Herkunft in der Vergangenheit eher Mobilitätserfahrungen gesammelt haben und diese die Wahrscheinlichkeit erneut mobil zu werden erhöhen. Die Ergebnisse einer ordinalen logistischen Regression zeigen außerdem, dass sowohl die soziale Herkunft als auch vorherige Mobilitätserfahrungen einen statistisch bedeutsamen Effekt auf den Stellensuchradius ausüben. Auf der Basis dieser empirischen Ergebnisse und suchtheoretischen Überlegungen wird argumentiert, dass die soziale Selektivität von Auslandsaufenthalten einen Beitrag zur Reproduktion sozialer Ungleichheit bei Hochqualifizierten liefert.

\section{Einleitung}

Ein wesentliches Ziel der im Rahmen des Bologna Prozesses durchgeführten Studiengangsreformen ist die Steigerung der internationalen Mobilität von Studierenden. Nicht zuletzt deshalb ist die internationale Mobilität von Studierenden und Hochqualifizierten wieder in den Mittelpunkt des Interesses der Fachöffentlichkeit gerückt. Im Rahmen dieser Debatte werden Hindernisse von räumlicher Mobilität während des Studiums insbesondere im ,workload“ und in der Struktur der neuen Studiengänge gesehen. Ein wesentlicher Einflussfaktor von räumlicher (Im-)Mobilität, der bei dieser Diskussion zu wenig Beachtung findet, ist die persönlichfamiliäre Situation der Studierenden und jungen Erwerbstätigen und dabei insbesondere deren soziale Herkunft. ${ }^{1}$

In diesem Artikel wird deshalb erstens der Einfluss der sozialen Herkunft auf die internationale Mobilität und den Stellensuchradius von Studierenden und Hochschulabsolventen theoretisch und empirisch untersucht, um darauf aufbauend zweitens die Rolle des herkunftsspezifisch unterschiedlichen Mobilitätsverhaltens für die Berufschancen von Hochqualifizierten zu diskutieren.

Während der Bildungskarriere müssen Akteure mehrere Mobilitätsentscheidungen treffen (vgl. Rolfes 1996). Nach dem Erwerb der Hochschulreife steht eine Entscheidung für einen Studienort an. Während des Studiums besteht die Möglichkeit einen Auslandsaufenthalt zu absolvieren und nach dem Studium wird eine Erwerbstätigkeit gesucht (vgl. Wagner 1989, Birg / Flöthmann 1992). Empirische Studien über das räumliche Mobilitätsverhalten im deutschen Bildungssystem kommen hier zu dem Ergebnis, dass räumliche Mobilität mit der sozialen Herkunft in einem engen Zusammenhang steht: Erstens besuchen Studienberechtigte

1 Generell kann im Hinblick auf die Forschungsliteratur festgehalten werden, dass trotz einiger Veröffentlichungen zu diesem Thema Wechselwirkungen zwischen räumlicher Mobilität und sozialer Ungleichheit noch nicht ausreichend erforscht wurden (vgl. Savage 1988; Abraham / Nisic 2007).

Soziale Welt 63 (2012), S. $45-64$ 
mit „nicht-akademischem Familienhintergrund“ eher eine Hochschule in der Nähe ihres Wohnortes als Studienberechtigte mit akademischem Familienhintergrund (vgl. Lörz 2008). Zweitens besteht ein Zusammenhang zwischen der sozialen Herkunft der Studierenden und der räumlichen Mobilität während des Studiums (Jahr et al. 2002; Isserstedt / Link 2008; Lörz / Krawietz 2011): Je höher der höchste Bildungsabschluss der Eltern, desto eher wird ein Studierender im Studium international mobil. Fabian und Minks (2008) kommen weiterhin zu dem Ergebnis, dass auch internationale Mobilität beim Berufseinstieg sozial selektiv ist und Absolventen mit einer höheren sozialen Herkunft eher eine erste Erwerbstätigkeit im Ausland antreten.

Empirische Mobilitätsstudien zeigen in diesem Zusammenhang allgemein, dass vorherige Mobilitätserfahrungen sowohl die Entfernung, in der nach einer Erwerbsstelle gesucht wird (Bailey 1993), als auch die Wahrscheinlichkeit erneut mobil zu werden erhöhen (Curran / Rivera-Fuentes 2003; Del Rey Poveda 2007; Diehl / Dixon 2005; Enders / Bornmann 2002; Massey 1987; Massey et al. 1987; Massey und Espinosa 1997; Massey / García Espana 1987).

Ein weiterer Forschungsstrang befasst sich mit dem Effekt der sozialen Herkunft auf die Bildungschancen (siehe bspw. Becker 2000; Blossfeld 1993; Henz / Maas 1995; Hillmert / Jacob 2005; Köhler 1992; Konietzka 1999; Krais 1996; Mayer 1991; Mayer und Blossfeld 1990; Meulemann 1995; Müller / Haun 1994; Rodax 1995; Schimpl-Neimanns 2000; Shavit / Blossfeld 1993). Diese Studien kommen zu dem Ergebnis, dass der größte Teil der sozialen Selektion beim Übergang in die Sekundarstufe und in das Hochschulsystem stattfindet. Die Entscheidungen, die an diesen Schwellen getroffen werden, haben tiefgreifende Konsequenzen für die Bildungs- und Erwerbskarriere. Doch auch nach dem Hochschulabschluss spielt die soziale Herkunft noch eine wichtige Rolle (Hartmann 2010; Hartmann / Kopp 2001). Hartmann (2002) untersucht die Besetzung von Führungspositionen und zeigt in diesem Zusammenhang, dass Bildungstitel zwar eine Vorauswahl bewirken, die endgültige Auswahl von Führungspersonal jedoch auch direkt mit der sozialen Herkunft der Hochschulabsolventen zusammenhängt. Die Karrierechancen von Hochschulabsolventen mit einer hohen sozialen Herkunft sind dementsprechend bei gleichen Bildungstiteln und Leistungssignalen besser. Dieser Vorsprung vergrößert sich zudem mit der Zeit im Erwerbsleben. Hartmann (2002) erklärt diesen Effekt durch das Habituskonzept von Bourdieu (1982). Demzufolge wissen Hochschulabsolventen „aus gutem Hause“ eher, wie man sich in Führungskreisen bewegt. Dieses inkorporierte kulturelle Kapital dient - bspw. bei Bewerbungsgesprächen - als Distinktionsmerkmal und hilft den Bewerbern dabei sich durchzusetzen.

Die beiden soeben resümierten Forschungsstränge sollen im Folgenden verbunden werden, indem räumliche Mobilität als vermittelnder Mechanismus gesehen wird, der Studierenden und Hochschulabsolventen mit hoher sozialer Herkunft im Verlauf der Bildungs- und Erwerbskarriere Vorteile verschafft. Dabei werden direkte und indirekte Effekte der sozialen Herkunft untersucht. Effekte der sozialen Herkunft, die bestehen bleiben, wenn auf Heterogenität kontrolliert wird, werden im Folgenden als direkte Effekte bezeichnet. Als indirekte Effekte der sozialen Herkunft werden mobilitätsfördernde Entscheidungen in der Vergangenheit bezeichnet, die von der sozialen Herkunft beeinflusst werden: Wenn junge Erwerbstätige mit hoher sozialer Herkunft schon beim Antritt eines Studiums und während des Studiums eher räumlich mobil sind und Erfahrungen mit räumlicher Mobilität die Bereitschaft zu weiteren Mobilitätsschritten erhöhen, dann liegt ein indirekter Effekt der sozialen Herkunft vor. In diesem Zusammenhang sollen folgende Forschungsfragen diskutiert werden:

In welcher Form wirkt sich die soziale Herkunft direkt und indirekt - über vermittelnde Faktoren - auf die Wahrscheinlichkeit aus, einen studienbezogenen Auslandsaufenthalt $\mathrm{zu}$ absolvieren? 
Welchen direkten und indirekten Einfluss hat die soziale Herkunft auf den Radius der Stellensuche?

Welchen direkten und indirekten Einfluss übt die soziale Herkunft auf die Wahrscheinlichkeit aus, den Berufseinstieg im Ausland zu vollziehen?

Um diese Forschungsfragen zu beantworten, werden im nächsten Abschnitt zunächst theoretische Überlegungen zu Determinanten und Konsequenzen internationaler Mobilität im Hinblick auf die soziale Herkunft präsentiert. Anschließend werden die Daten des Bayerischen Absolventenpanels beschrieben, die bei den empirischen Auswertungen herangezogen werden. Darauf aufbauend werden binäre und ordinale logistische Regressionsanalysen dargestellt, mit denen die Daten analysiert werden. In Abschnitt 4 . werden dann die empirischen Ergebnisse vorgestellt. Der Artikel schließt mit einer Zusammenfassung der empirischen Ergebnisse und einer Diskussion der Implikationen für den langfristigen Berufserfolg von Hochqualifizierten.

\section{Theorie: Räumliche Mobilität als Investition in Humankapital}

Die Humankapitaltheorie konzipiert Migration als eine Investition, die Kosten beinhaltet und einen Ertrag erbringt. Im Falle von Studierenden und Hochschulabsolventen sind die Erträge bspw. in einem speziellen Kompetenzerwerb an einer ausländischen Hochschule oder einer besseren erwarteten Verwertbarkeit des eigenen Humankapitals am Zielort zu sehen (vgl. Faggian / McCann 2009: 32). Gemäß dem Humankapitalmodell wird ein Studierender oder ein Hochschulabsolvent mobil, wenn der erwartete Ertrag der Migration deren Kosten übersteigt (siehe bspw. DaVanzo 1976; Speare 1971, 1974). Kosten und Erträge können sowohl monetäre als auch nicht-monetäre Elemente (z.B. Informationskosten und psychische Kosten) beinhalten (DaVanzo 1976; Sjastaad 1962).

Wird dieses theoretische Modell auf den Einfluss der sozialen Herkunft auf das Mobilitätsverhalten im Studium, den Stellensuchradius und das Mobilitätsverhalten beim Berufseinstieg von Studierenden und Hochschulabsolventen bezogen, lassen sich folgende Zusammenhänge diskutieren.

\section{Finanzielle Unterstützung}

Für Studierende und Berufseinsteiger mit hoher sozialer Herkunft ist es leichter, die Kosten für einen Umzug aufzubringen, da die Wahrscheinlichkeit hier höher ist, dass die Eltern finanziell „unter die Arme greifen“ können. Bei Studierenden und Berufseinsteigern mit eher ungünstigen sozialen Hintergrundcharakteristiken können außerdem zusätzliche Kosten in Form von Zinsen anfallen, wenn zur Aufbringung der Mobilitätskosten ein Darlehen aufgenommen werden muss.

\section{Psychologische Unterstützung}

Außerdem unterstützen Eltern mit günstigen sozialen Charakteristiken den Schritt ihrer Sprösslinge ins Ausland zu gehen auch moralisch eher (siehe Pineda 2008).

\section{Erfahrungen mit räumlicher Mobilität}

Studierende und Hochschulabsolventen, deren Eltern einen hohen sozialen Status aufweisen, haben eher in der Vergangenheit schon Erfahrungen mit räumlicher Mobilität gesammelt. Gemäß DaVanzo $(1976,1983)$ reduzieren Erfahrungen mit räumlicher Mobilität die Kosten weiterer Mobilitätsschritte - bspw. aufgrund räumlich disperserer Freundschaftsnetzwerke (siehe auch Ohnmacht et al. 2008) - woraus geringere Mobilitätskosten und eine gesteigerte 
Mobilitätsneigung resultieren. Dementsprechend können folgende Hypothesen formuliert werden:

H1 a: Es besteht ein positiver Zusammenhang zwischen einer hohen sozialen Herkunft und der Wahrscheinlichkeit während des Studiums ins Ausland zu gehen.

H1 b: Es besteht ein positiver Zusammenhang zwischen einer hohen sozialen Herkunft und dem Stellensuchradius beim Berufseinstieg.

H1 c: Es besteht ein positiver Zusammenhang zwischen einer hohen sozialen Herkunft und der Wahrscheinlichkeit, den Berufseinstieg im Ausland zu vollziehen.

Beim Studienantritt muss die Entscheidung getroffen werden, ob in der Heimatregion, in einem anderen Bundesland oder im Ausland studiert wird. Analog besteht während des Studiums die Entscheidungssituation darin, an der Universität, an der das Studium begonnen wurde, zu bleiben, an eine andere Universität im selben Nationalstaat zu wechseln oder international mobil zu werden.

Beim Berufseinstieg können Hochschulabsolventen eine Stellensuche in der Region, in der die Eltern leben, oder in der Universitätsregion durchführen. Alternativ kann der Stellensuchradius sowohl national als auch international ausgeweitet werden. Analog dazu kann ein Hochschulabsolvent den Berufseinstieg in der Region des Wohnortes der Eltern, der Hochschulregion oder einer anderen nationalen oder internationalen Region vollziehen. Gemäß der Argumentation von DaVanzo $(1976,1983)$ wird jeder Mobilitätsschritt an einer dieser „Schwellen“ weitere räumliche Mobilität wahrscheinlicher werden lassen (siehe auch Faggian et al. 2007 a: 523).

H2 a: Studierende, die ihre Hochschulzugangsberechtigung außerhalb von Bayern erworben haben, gehen während dem Studium eher ins Ausland.

H2 b: Studierende, die ihre Hochschulzugangsberechtigung außerhalb von Bayern erworben haben, weisen einen größeren Stellensuchradius auf.

H2 c: Studierende, die ihre Hochschulzugangsberechtigung außerhalb von Bayern erworben haben, akzeptieren eher ein Jobangebot aus dem Ausland und vollziehen infolgedessen eher den Berufseinstieg im Ausland.

H2 d: Auslandserfahrungen während des Studiums gehen mit einem größeren Stellensuchradius einher.

H2 e: Auslandserfahrungen während des Studiums gehen mit einer größeren Wahrscheinlichkeit internationaler Mobilität beim Berufseinstieg einher.

Außerdem postuliert das mikroökonomische Humankapitalmodell, dass räumliche Mobilität von den folgenden Faktoren beeinflusst wird: Alter (bspw. Speare 1974), Partnerschaftsstatus (siehe auch Abraham et al. 2010; Auspurg / Abraham 2007; Mincer 1978) und Fremdsprachenkenntnisse sowie Nationalität (Chiswick 1991). Diese Variablen werden deshalb in den empirischen Analysen als Kontrollvariablen verwendet.

Abschließend sollen nun Implikationen einer höheren Mobilitätsbereitschaft für den Berufserfolg von Hochschulabsolventen theoretisch diskutiert werden. Hierzu wird auf eine Kombination des oben diskutierten mikroökonomischen Humankapitalmodells und suchtheoretischen Überlegungen zurückgegriffen (siehe hierzu auch Faggian / McCann 2009; Molho 1986).

In Suchtheorien wird angenommen, dass Akteure ein optimales Stellensuchverhalten an den Tag legen. Demzufolge hängt die Länge der Jobsuche einerseits von der Einkommensverteilung ab, die ein Absolvent durch seine Kompetenzen und Merkmalskombination fordern kann und andererseits von den Kosten, weitere Jobangebote zu generieren (vgl. Simpson 1992). Nach diesem Ansatz hört ein Absolvent auf nach weiteren Jobs zu suchen, wenn das Einkommen eines Jobangebots mindestens gleich seinem Reservationslohn ist (vgl. Faggian / McCann 2009). Der Reservationslohn ist wiederum erreicht, wenn bei einem Jobangebot die marginalen 
Kosten, ein weiteres Jobangebot zu generieren, gleich sind wie die marginalen Erträge, wenn die Jobsuche fortgesetzt würde (vgl. Herzog et al. 1985; Molho 1986).

Werden nun diese suchtheoretischen Überlegungen mit dem humankapitaltheoretischen Migrationsmodell kombiniert, lässt sich folgern, dass sowohl Verringerungen der Mobilitätskosten als auch reduzierte Suchkosten (durch räumlich dispersere Informationen über Stellenangebote und einen größeren Suchradius) mit verlängerten Suchdauern, höheren Reservationslöhnen und höheren Mobilitätserträgen einhergehen (vgl. Faggian / McCann 2009: 32). Aus diesem Modell folgt also ein Zusammenhang zwischen Mobilitätserfahrungen und weiterer räumlicher Mobilität einerseits und - aufgrund der reduzierten Mobilitäts- und Suchkosten - der langfristigen Einkommensentwicklung von Hochschulabsolventen andererseits (vgl. Faggian et al. 2007 b). Dieser Zusammenhang sollte unabhängig von unterschiedlichen lokalen Opportunitätsstrukturen (DaVanzo 1978) und Humankapitalunterschieden von Hochschulabsolventen gelten (vgl. Faggian et al. 2007 b).

\section{Daten und Methode}

Die Hypothesen werden anhand der Daten der ersten Welle des Bayerischen Absolventen Panels $(B A P)$ der 2005/06er Abschlusskohorte empirisch geprüft. ${ }^{2}$ Die Grundgesamtheit bilden die Diplom- und Magisterabsolventen aller Fächergruppen, die bayernweit in diesem Prüfungsjahrgang mindestens 50 Absolventen aufweisen. Zur Datengewinnung wurde eine Vollerhebung durchgeführt, bei der die Absolventen von 26 bayerischen Hochschulen mittels eines schriftlichen Fragebogens entweder postalisch oder online befragt wurden. In dieser Befragungswelle wurde neben den üblichen Fragen zum Studienverlauf, dem Stellensuchprozess und dem Berufseinstieg ein umfassender Themenkomplex zur räumlichen Mobilität implementiert.

Repräsentativitätsprüfungen ergaben keine systematischen Verzerrungen aufgrund von selektivem Rücklauf in Bezug auf Studienfach, Hochschule oder Geschlecht. ${ }^{3}$ Bei Analysen räumlicher Mobilität ist aber davon auszugehen, dass insbesondere international mobile Personen systematisch unterrepräsentiert sind. Mobile Personen sind schwerer auffindbar und die Zustellung der Fragebögen ins Ausland ist mit deutlich mehr Hindernissen verbunden als der postalische Versand innerhalb Deutschlands. Diesem Problem wurde durch die Kombination von online und postalischer Befragung begegnet. Nichtsdestotrotz bleibt festzuhalten, dass der Anteil der mobilen Personen wahrscheinlich unterschätzt wird. Hypothesen über theoretisch begründete Zusammenhänge können mit den Daten anhand multivariater Modelle dennoch

2 Das Bayerische Absolventenpanel (BAP) ist eine landesweite, repräsentative und langfristig angelegte Absolventenstudie für eine breite Auswahl an Studienfächern. Ziel ist es, Informationen zur Ausbildungsqualität der Hochschulen, zum Übergang der Absolventen in den Arbeitsmarkt und zur weiteren beruflichen Laufbahn zu gewinnen. Die wissenschaftliche Leitung dieses Langzeitprojekts liegt beim Bayerischen Staatsinstitut für Hochschulforschung und Hochschulplanung (IHF). Die Befragungen werden zusammen mit den bayerischen Hochschulen durchgeführt. Für das BAP werden ausgewählte Absolventenjahrgänge zu mehreren Zeitpunkten befragt: ein erstes Mal rund anderthalb Jahre nach ihrem Hochschulabschluss, ein zweites Mal nach fünf und ein drittes Mal nach neun Jahren. Bislang sind bei drei Erhebungen die Absolventenjahrgänge 2003 / 04 (1. und 2. Welle) und 2005 / 06 (nur 1.Welle) untersucht worden.

3 Die Darstellung zum Datensatz stammt im Wesentlichen aus dem methodischen Anhang der Hochschulberichte und können auf der IHF Homepage unter folgendem Link nachgelesen werden: http:// www.ihf.bayern.de/?BAP\%3A_Das_Bayerische_Absolventenpanel:Ergebnisse:Hochschulberichte. Der gesamte Fragebogen des Bayerischen Absolventenpanels des Jahres 2005/06 kann im Internet unter der Adresse: http://www.ihf.bayern.de/?Bayerisches_Absolventenpanel_\%28BAP\%29 heruntergeladen werden. 
sehr gut getestet werden. Tabelle 1 zeigt eine Übersicht zu Fallzahlen und deskriptiven Werten der verwendeten Variablen.

Bei den statistischen Analysen gehen unterschiedliche Fallzahlen in die Modelle ein. Bei den Determinanten eines studienbezogenen Auslandsaufenthalts sind es 5613 Beobachtungen, bei der Analyse der Determinanten der ersten Erwerbstätigkeit im Ausland 4991. Der Unterschied ist im Wesentlichen darauf zurückzuführen, dass bei Letzteren nur die Absolventen berücksichtigt werden, die bereits eine erste Erwerbstätigkeit aufgenommen haben. Bei den Modellen zur Entfernung der Stellensuche gehen schließlich 3841 Akteure in die Untersuchung ein: Absolventen, die ohne Suche eine Stelle angeboten bekommen oder aus anderen Gründen keine Stelle gesucht haben, bleiben unberücksichtigt.

Als abhängige Variablen gehen die dichotomen Variablen ,studienbezogener Auslandsaufenthalt" $1=$ ja, $0=$ nein sowie ,erste Erwerbstätigkeit im Ausland“ $=1$, ,erste Erwerbstätigkeit in Deutschland" $=0$ in die Mobilitätsanalysen ein. Der Stellensuchradius wurde durch drei hierarchische Kategorien abgebildet: 1=regionale Stellensuche, 2=nationale Stellensuche und $3=$ internationale Stellensuche.

Zur Operationalisierung des Status der Eltern wurde sowohl die aktuelle berufliche Position der Mutter als auch des Vaters berücksichtigt, um eine möglichst gute Näherung des tatsächlichen Haushaltseinkommens der Herkunftsfamilie zu erreichen: Wenn beide Elternteile einen hohen sozioökonomischen Status aufweisen, wird ihnen ein homogen hoher Status zugeordnet. ${ }^{4}$ Weist einer der beiden Elternteile einen hohen und der andere einen niedrigen Status auf, wurde der Status als heterogen klassifiziert. Ein homogen niedriger Status liegt vor, wenn beide Elternteile einen niedrigen sozioökonomischen Status aufweisen.

Mobilitätserfahrungen werden durch Dummyvariablen operationalisiert. Studierende bzw. Hochschulabsolventen, die ihre Hochschulzugangsberechtigung in einem der übrigen Bundesländer erwarben und Akteure, die diese im Ausland absolvierten, gehen getrennt in die Analysen ein. Bei den Modellen zum Stellensuchradius und der internationalen Mobilität beim Berufseinstieg wird außerdem berücksichtigt, ob ein studienbezogener Auslandsaufenthalt absolviert wurde.

Weiterhin werden das Geschlecht und die Nationalität (als Dummyvariablen) sowie das Alter (als metrische Variable) in allen Modellen als Kovariablen berücksichtigt. ${ }^{5}$ Bei den Modellen zum Stellensuchradius und dem Berufseinstieg im Ausland gehen außerdem der Beziehungsstatus (zum Zeitpunkt der Befragung) und die Fremdsprachenkompetenz zum Zeit-

4 Folgende berufliche Stellungen wurden als hoch klassifiziert: Leitender Angestellter (z.B. Abteilungsleiter, Direktor), wissenschaftlich qualifizierter Angestellter mit mittlerer Leitungsfunktion (z.B. Projekt-, Gruppenleiter), selbständiger in freien Berufen, selbständiger Unternehmer, Beamter im höheren Dienst, Beamter im gehobenen Dienst, wissenschaftlicher Angestellter ohne Leitungsfunktion. Als niedrige berufliche Stellung wurden folgende Zustände zusammengefasst: Nie erwerbstätig gewesen, mithelfender Familienangehöriger, un- / angelernter Arbeiter, Facharbeiter mit Lehre, Beamter im einfachen / mittleren Dienst, ausführender Angestellter (z.B. Verkäufer, Schreibkraft). Qualifizierte Angestellte (z.B. Sachbearbeiter) wurden als hohe berufliche Stellung klassifiziert, wenn gleichzeitig ein Hochschulabschluss vorlag und sonst als niedrige berufliche Stellung.

5 Deskriptive Analysen bezüglich des Alters der Befragten zum Zeitpunkt des Studienabschlusses zeigten, dass zwei Seniorenstudierende im Alter von 70 und 71 Jahren in der Stichprobe vertreten waren. Weiterhin waren 57 Personen in der Stichprobe, die zum Zeitpunkt des Studienabschlusses älter als 40 waren. Die Modelle wurden zunächst für alle Untersuchungseinheiten geschätzt. In einem zweiten Schritt wurden alle über 40-Jährigen ausgeschlossen. Die Ergebnisse der Tabellen, die jetzt dargestellt sind, beruhen auf Schätzungen, bei denen die beiden Personen im Rentenalter ausgeschlossen wurden. Die Ergebnisse der Schätzungen unter Einbeziehung unterschiedlicher Altersgruppen erwiesen sich dabei qualitativ als völlig robust. 
punkt des Studienabschlusses in die Analysen ein. ${ }^{6}$ Bei der Fremdsprachenkompetenz wurden drei Kategorien gebildet (gute, mittlere und schlechte Fremdsprachenkompetenz), von denen die ersten beiden Kategorien als Dummyvariablen in die multivariaten Analysen eingehen.

Da der Schwerpunkt dieser Untersuchung nicht auf der Analyse des Einflusses unterschiedlicher Studiengangsprofile oder der expliziten Untersuchung von Hochschulmerkmalen liegt, werden unterschiedliche Fächergruppen und Hochschulen durch Dummyvariablen kontrolliert. ${ }^{7}$ Dabei wurden fachspezifische deskriptive Auswertungen der Mobilitätswahrscheinlichkeit (hier nicht aufgeführt) dazu verwendet, um Studienfachgruppen zu bilden, bei denen möglichst keine Studienfächer mit hoch mobilen und besonders sesshaften Studierenden bzw. Absolventen zusammengeworfen werden. ${ }^{8}$

Zur Schätzung der internationalen Mobilität während des Studiums und beim Berufseinstieg wurden binäre logistische Regressionsmodelle herangezogen (siehe bspw. Tutz 2000; Menard 2002 oder Rindskopf 2004). Zur Schätzung der Determinanten des Stellensuchradius wurde eine ordinale logistische Regression durchgeführt (vgl. Long / Freese 2006). Bei der Schätzung eines solchen Modells mit drei Merkmalsausprägungen der abhängigen Variable wird angenommen, dass der Effekt eines Anstiegs einer erklärenden Variable um eine Einheit den gleichen Effekt hat, unabhängig davon, ob die abhängige Variable zwischen der ersten und der zweiten Kategorie oder der zweiten und der dritten Kategorie dichotomisiert wird (vgl. Long / Freese 2006; Rabe-Hesketh / Skrondal 2008; Kühnel / Krebs 2010). ${ }^{9}$ Zur Überprüfung dieser Annahme wurde erstens ein Wald Test durchgeführt (vgl. Long / Freese 2006) und zweitens wurden zwei getrennte binäre logistische Regressionen für die beiden Übergänge geschätzt. Für einzelne unabhängige Variablen musste die Annahme proportionaler Chancen verworfen werden. Dies wird bei der Interpretation der Ergebnisse berücksichtigt. Weiterhin werden die Effekte in allen Modellen als averaged marginal effects (AME) dargestellt, weil diese Effekte geeignet sind, um Koeffizienten von schrittweise aufgebauten logistischen Regressionsmodellen miteinander zu vergleichen (vgl. Best / Wolf 2010; Mood 2010). Ein AME kann dabei folgendermaßen interpretiert werden:

„In words, AME expresses the average effect of $x_{1}$ on $P(y=1)$. It does so by taking the logistic $P D F$ [probability distribution function] at each observation's estimated logit, multiplying this by the coefficient for $x_{1}$, and averaging this product over all observations." (Mood 2010: 75)

Außerdem wurden in den empirischen Auswertungen robuste Standardfehler berechnet. ${ }^{10}$

6 Bei Ausländern kann nicht zwischen Bildungsinländern und anderen Ausländern unterschieden werden. Der Schätzwert für Singles ist eher ein approximativer Schätzer, da eineinhalb Jahre nach dem Studienabschluss befragt wurde. Die theoretisch interessante Variable wäre der Partnerschaftsstatus zum Zeitpunkt der Mobilitätsentscheidung. Wer nach dem Studium ins Ausland ging und danach einen Partner fand, wird also fälschlicherweise als gebunden eingestuft. Ebenso werden Absolventen, deren Beziehung an dem Arbeitsplatz im Ausland zerbrach, fälschlicherweise als Singles eingestuft.

7 Für Diskussionen, wann Modelle mit zufälligen Effekten und wann Modelle mit fixen Effekten verwendet werden sollten, um eine hierarchische Datenstruktur zu modellieren siehe bspw. Snijders / Bosker (1999); Blien / Wiedenbeck (2002); Wooldridge (2003) oder Brüderl (2010). Die Schätzer von Modellen mit fixen Effekten und die hier gewählte Modellierung über Dummyvariablen sind unter bestimmten Annahmen identisch (vgl. Wooldridge 2002: 273). Wenn wie hier nicht-lineare Regressionsmodelle betrachtet werden, führt die Dummy-Methode zwar nicht zu konsistenten Schätzungen, Simulationsstudien haben in diesem Zusammenhang jedoch gezeigt, dass Verzerrungen gering sind, wenn auf Ebene 2 mehr als 10 Beobachtungen vorliegen (vgl. Brüderl 2010: 986).

8 Für eine Analyse des Mobilitätsverhaltens unterschiedlicher Studienfächer siehe Falk / Kratz (2009).

9 Diese Annahme wird auch als proportional odds assumption bezeichnet.

10 Für methodische Details hierzu siehe Angrist / Pischke 2009: Kap. 8. 
Tabelle 1: Übersicht über deskriptive Kennwerte der Stichprobe

\begin{tabular}{|c|c|c|c|c|c|c|}
\hline & $\begin{array}{l}\text { Anzahl } \\
\text { gültiger } \\
\text { Fälle }\end{array}$ & Min. & Max. & Mittelwert & $\begin{array}{l}\text { Stand.- } \\
\text { abw. }\end{array}$ & Median \\
\hline $\begin{array}{l}\text { Studienbezogener Auslandsaufent- } \\
\text { halt }(1=\mathrm{ja})\end{array}$ & 6354 & 0 & 1 & 0,40 & - & - \\
\hline $\begin{array}{l}\text { Stellensuchradius } \\
(1=\text { regional, } 2=\text { bundesweit, } 3=\text { in- } \\
\text { ternational })\end{array}$ & 4380 & 1 & 3 & 1,64 & - & - \\
\hline $\begin{array}{l}\text { Erste Erwerbstätigkeit im Ausland } \\
(1=\mathrm{ja})\end{array}$ & 5599 & 0 & 1 & 0,06 & - & - \\
\hline \multicolumn{7}{|l|}{$\begin{array}{l}\text { Status Eltern } \\
\text { Ref. (homogen niedrig) }\end{array}$} \\
\hline Status heterogen $(1=\mathrm{ja})$ & 5800 & 0 & 1 & 0,47 & - & - \\
\hline Status homogen hoch $(1=\mathrm{ja})$ & 5800 & 0 & 1 & 0,2 & - & - \\
\hline $\begin{array}{l}\text { Ort des Erwerbs der Hochschulzu- } \\
\text { gangsberechtigung } \\
\text { (Ref.: Bayern) }\end{array}$ & & & & & - & - \\
\hline $\begin{array}{l}\text { Hochschulzugangsberechtigung } \\
\text { aus einem anderen Bundesland }\end{array}$ & 6121 & 0 & 1 & 0,20 & - & - \\
\hline $\begin{array}{l}\text { Hochschulzugangsberechtigung aus } \\
\text { dem Ausland }\end{array}$ & 6121 & 0 & 1 & 0,02 & - & - \\
\hline Beziehungsstatus ( $1=$ Single) & 6179 & 0 & 1 & 0,32 & - & - \\
\hline Geschlecht (1=weiblich) & 6248 & 0 & 1 & 0,47 & - & - \\
\hline Alter & 6176 & 22 & 63 & 27,88 & 2.91 & 27 \\
\hline \multicolumn{7}{|l|}{$\begin{array}{l}\text { Fremdsprachenkompetenz } \\
\text { (Ref.: schlechte Fremdsprachen- } \\
\text { kenntnisse) }\end{array}$} \\
\hline $\begin{array}{l}\text { Mittlere Fremdsprachenkenntnisse } \\
(1=\mathrm{ja})\end{array}$ & 6247 & 0 & 1 & 0,15 & - & - \\
\hline $\begin{array}{l}\text { Gute Fremdsprachenkenntnisse } \\
(1=\mathrm{ja})\end{array}$ & 6247 & 0 & 1 & 0,66 & - & - \\
\hline Ausländer (1=ja) & 6199 & 0 & 1 & 0,03 & - & - \\
\hline
\end{tabular}

\section{Empirische Auswertungen}

Die empirischen Resultate werden nun folgendermaßen dargestellt: Zunächst werden Determinanten eines studienbezogenen Auslandsaufenthalts präsentiert, um anschließend Einflussfaktoren auf die Entfernung der Stellensuche zu analysieren. In Abschnitt 4.3 werden dann die Determinanten einer ersten Erwerbstätigkeit im Ausland präsentiert. Bei den multivariaten Auswertungen wird stets in Modell 1 der Effekt der sozialen Herkunft geschätzt, ohne dabei auf relevante Drittvariablen zu kontrollieren. In Modell 2 werden dann vorherige Mobilitätserfahrungen einbezogen. Dieses Vorgehen wurde gewählt, da aufgrund der theoretischen Ausführungen erwartet wird, dass der indirekte Effekt der sozialen Herkunft insbesondere durch vorherige Mobilitätserfahrungen wirkt. Modell 3 untersucht schließlich, ob die Effekte der sozialen Herkunft und der vorherigen Mobilitätserfahrungen robust bleiben, wenn auf biografische Merkmale sowie Studienfach- und Hochschuldummies kontrolliert wird. 


\subsection{Auslandserfahrungen während des Studiums}

Tabelle 2 zeigt die Schätzergebnisse eines logistischen Regressionsmodells. In Modell 1 wird nur der berufliche Status der Eltern berücksichtigt. Hier zeigt sich ein höchst signifikanter Effekt: ${ }^{11}$ Studierende, bei denen beide Elternteile eine hohe berufliche Stellung innehaben, gehen demzufolge signifikant wahrscheinlicher während des Studiums ins Ausland. Bei Studierenden, bei denen nur ein Elternteil eine berufliche Stellung mit hohem Status aufweist, ist der Effekt schwächer, bleibt aber hoch signifikant.

Wenn auf vorherige Mobilitätserfahrungen kontrolliert wird (Modell 2), zeigt sich, dass Studierende mit einer Hochschulzugangsberechtigung in einem anderen Bundesland während des Studiums signifikant wahrscheinlicher ins Ausland gehen, während Studierende, die ihre Hochschulzugangsberechtigung im Ausland erworben haben, keine erhöhte Wahrscheinlichkeit aufweisen, einen studienbezogenen Auslandsaufenthalt zu absolvieren.

Die Effektstärke der sozialen Herkunft schwächt sich in diesem Modell nur unwesentlich ab und bleibt höchst signifikant.

In Modell 3 werden weitere bedeutsame Einflussfaktoren kontrolliert. Das Geschlecht zeigt dabei keinen statistisch bedeutsamen Einfluss. Vom Alter geht der vom mikroökonomischen Humankapitalmodell prognostizierte Effekt aus: Je älter ein Studierender ist, desto geringer ist die Wahrscheinlichkeit eines studienbezogenen Auslandsaufenthalts. Bei den Studienfächern zeigt sich, dass Studierende der Wirtschaftswissenschaften am ehesten einen studienbezogenen Auslandsaufenthalt absolvieren, gefolgt von den Sprach- und Kulturwissenschaften.

Der Effekt der sozialen Herkunft schwächt sich in Modell 3 ab, bleibt aber hoch signifikant. Diese Ergebnisse zeigen, dass ein statistisch bedeutsamer Zusammenhang von der sozialen Herkunft auf die Wahrscheinlichkeit während des Studiums ins Ausland zu gehen besteht, auch wenn auf persönliche, studienfachspezifische und hochschulspezifische Unterschiede kontrolliert wird. Der indirekte Effekt der sozialen Herkunft - über eine höhere Wahrscheinlichkeit räumlicher Mobilität in der Vergangenheit - ist bei dieser Mobilitätsentscheidung eher gering, da sich der Effekt der sozialen Herkunft in Modell 2 nur unwesentlich abschwächt.

Tabelle 2: Determinanten eines studienbezogenen Auslandsaufenthalts, logistische Regression, averaged marginal effects

\begin{tabular}{lccc}
\hline & Modell 1 & Modell 2 & Modell 3 \\
\hline (Ref. Status homogen niedrig) & $0,176^{* * *}$ & $0,160^{* * *}$ & $0,097^{* * *}$ \\
Status homogen hoch & $(0,017)$ & $(0,076)$ & $(0,017)$ \\
& & & \\
Status heterogen & $0,073^{* * *}$ & $0,069^{* * *}$ & $0,035^{*}$ \\
& $(0,016)$ & $(0,016)$ & $(0,014)$
\end{tabular}

(Ref. HZB in Bayern)

11 Ob bei Vollerhebungen Signifikanztests sinnvoll interpretiert werden können ist in der Literatur umstritten (Behnke 2005). Wenn angenommen wird, dass der Non-Response zufällig ist, dann liegt eine Zufallsstichprobe vor und unter Anwendung von Signifikanztests kann auf die Grundgesamtheit bayerischer Hochschulabsolventen geschlossen werden. Wenn außerdem Hypothesen über theoretische Ursache-Wirkungszusammenhänge - anhand einer durch eine Vollerhebung gewonnenen Auswahl an Untersuchungseinheiten - getestet werden sollen, kann die Anwendung statistischer Signifikanztests ebenfalls gerechtfertigt werden, weil dann das Sample als exemplarische Stichprobe aller Hochqualifizierten gesehen werden kann (vgl. Broscheid/Gschwend 2005). 
HZB in Restdeutschland

$0,181^{* * *}$

$(0,014)$

$-0,017$

$(0,046)$

Frau

$-0,005$

$(0,013)$

$-0,012^{* * *}$

$(0,002)$

(Ref. Informatik)

Kunst und Innenarchitektur

$0,205^{* * *}$

$(0,044)$

Sprach- und Kulturwissenschaften

$0,269^{* * *}$

$(0,036)$

Psychologie / Pädagogik / Soziologie /

$0,084^{*}$

Politikwissenschaften

$(0,035)$
$-0,123^{* *}$

$(0,047)$

$0,329^{* * *}$

$(0,025)$

$0,193^{* * *}$

$(0,031)$

$0,105^{*}$

$(0,043)$

$0,102^{* *}$

$(0,031)$

Elektrotechnik / Maschinenbau

$0,163^{* * *}$

$(0,040)$

5613

Beobachtungen

5613

5613

$717,72^{* * *}$

Wald chi2

$102,87^{* * *}$

$229,95^{* * *}$

Robuste Standardfehler in Klammern; Modell 3 enthält 25 Hochschuldummies (Effekte nicht dargestellt); ${ }^{*} p<0,05 ;{ }^{* *} p<0,01 ;{ }^{* * *} p<0,001$ 


\subsection{Stellensuchradius}

Zur Analyse der Determinanten des Stellensuchradius wurde eine ordinale logistische Regression geschätzt (siehe Tabelle 3). Hier zeigt sich, dass Absolventen mit einer homogen hohen sozialen Herkunft in einer größeren Entfernung nach Stellen suchen. Dieser Effekt erweist sich in allen drei Modellen als statistisch hoch signifikant.

Auch von den vorherigen Mobilitätserfahrungen gehen weitgehend die theoretisch erwarteten Effekte aus: Eine Hochschulzugangsberechtigung in einem anderen Bundesland und ein studienbezogener Auslandsaufenthalt gehen mit einem größeren Stellensuchradius einher. Weiterführende Analysen zeigen in diesem Zusammenhang, dass Absolventen, die ihre Hochschulzugangsberechtigung in einem anderen Bundesland erworben haben, vor allem deutschlandweit nach Erwerbsstellen suchen. ${ }^{12}$ Absolventen, die einen studienbezogenen Auslandsaufenthalt absolviert haben, suchen sowohl eher deutschlandweit als auch eher international nach einer Stelle. ${ }^{13}$ Absolventen, die ihre Hochschulzugangsberechtigung im Ausland erworben haben, weisen hingegen keinen signifikant größeren Stellensuchradius auf.

Bei den Kontrollvariablen zeigt sich, dass Singles signifikant eher in einem größeren Umkreis suchen und dass die Bereitschaft, in einer größeren Entfernung nach Stellen zu suchen, mit dem Alter (nicht signifikant) abnimmt. Interessant ist auch, dass Frauen einen statistisch bedeutsam geringeren Suchradius aufweisen als Männer. ${ }^{14}$ Absolventen mit guten Fremdsprachenkenntnissen suchen ebenfalls in einer größeren Entfernung nach einer Stelle, was hauptsächlich durch eine verstärkte internationale Stellensuche zu erklären ist.

Bei den Studienfächern weisen die Kunst- und Innenarchitekten, Mathematiker und Naturwissenschaftler sowie die Architekten und Bauingenieure den größten Stellensuchradius auf.

Es besteht folglich ein statistisch bedeutsamer Zusammenhang zwischen der sozialen Herkunft und dem Stellensuchradius, auch wenn auf persönliche, studienfachspezifische und hochschulspezifische Unterschiede kontrolliert wird. Außerdem zeigen sich hier indirekte über vorherige Mobilitätserfahrungen vermittelte - Effekte. Dementsprechend verringert sich der Effekt eines homogen hohen Status um ca. 45\%, wenn (in Modell 2) auf vorherige Mobilitätserfahrungen kontrolliert wird.

$12 \mathrm{Ob}$ diese verstärkte deutschlandweite Stellensuche hauptsächlich durch die Absicht an den „Herkunftsort" zurückzuziehen verursacht wird, kann mit den vorhandenen Daten nicht untersucht werden.

13 Diese weiterführenden Analysen wurden im Rahmen der Überprüfung der ,proportional-odds-assumption" der Ordered-Logit-Regression durchgeführt (vgl. Long / Freese 2006; Rabe-Hesketh / Skrondal 2008).

14 Diesem Effekt wird anhand der 2.Welle der BAP-Befragung weiter nachgegangen werden. In diesen Daten werden Berufs- und Mobilitätsverläufe der ersten fünf Jahre nach Studienabschluss erfasst. Dies ermöglicht eine tiefergehende Untersuchung der Wechselwirkungen zwischen dem Geschlecht, der Fertilität, dem Mobilitätsverhalten und dem Berufserfolg. 
Tabelle 3: Determinanten der Stellensuchdistanz, ordinale logistische Regression, averaged marginal effects

\begin{tabular}{|c|c|c|c|}
\hline & Modell 1 & Modell 2 & Modell 3 \\
\hline \multicolumn{4}{|l|}{ (Ref. Status homogen niedrig) } \\
\hline Status homogen hoch & $\begin{array}{l}0,140^{* * *} \\
(0,020)\end{array}$ & $\begin{array}{l}0,077^{* * *} \\
(0,020)\end{array}$ & $\begin{array}{l}0,057^{* *} \\
(0,019)\end{array}$ \\
\hline Status heterogen & $\begin{array}{l}0,057^{*} \\
(0,018)\end{array}$ & $\begin{array}{c}0,030 \\
(0,017)\end{array}$ & $\begin{array}{c}0,029 \\
(0,016)\end{array}$ \\
\hline studienbezogener Auslandsaufenthalt & & $\begin{array}{l}0,211^{* * *} \\
(0,014)\end{array}$ & $\begin{array}{c}0,160^{* * *} \\
(0,015)\end{array}$ \\
\hline \multicolumn{4}{|l|}{ (Ref. HZB in Bayern) } \\
\hline HZB in Restdeutschland & & $\begin{array}{l}0,161^{* * *} \\
(0,016)\end{array}$ & $\begin{array}{c}0,110^{* * *} \\
(0,016)\end{array}$ \\
\hline HZB im Ausland & & $\begin{array}{c}0,078 \\
(0,061)\end{array}$ & $\begin{array}{c}0,070 \\
(0,069)\end{array}$ \\
\hline Single & & & $\begin{array}{c}0,141^{* * *} \\
(0,014)\end{array}$ \\
\hline Frau & & & $\begin{array}{c}-0,094^{* * *} \\
(0,016)\end{array}$ \\
\hline Alter & & & $\begin{array}{l}-0,004 \\
(0,003)\end{array}$ \\
\hline \multicolumn{4}{|l|}{ (Ref. schlechte Fremdsprachenk.) } \\
\hline sehr gute Fremdsprachenkompetenz & & & $\begin{array}{c}0,136^{* * *} \\
(0,028)\end{array}$ \\
\hline mittlere Fremdsprachenkompetenz & & & $\begin{array}{l}0,045^{*} \\
(0,019)\end{array}$ \\
\hline Ausländer & & & $\begin{array}{c}0,016 \\
(0,062)\end{array}$ \\
\hline \multicolumn{4}{|l|}{ (Ref. Informatik) } \\
\hline Kunst und Innenarchitektur & & & $\begin{array}{c}0,286^{* * *} \\
(0,049)\end{array}$ \\
\hline Sprach- und Kulturwissenschaften & & & $\begin{array}{l}0,146^{* *} \\
(0,046)\end{array}$ \\
\hline
\end{tabular}


Psychologie / Pädagogik / Soziologie / $\quad 0,203^{* * *}$

Politikwissenschaften $\quad(0,040)$

$\begin{array}{lr}\text { Soziale Arbeit } & 0,076\end{array}$

Wirtschaftswissenschaften $\quad 0,136^{* * *}$

Mathematik und Naturwissenschaften $\quad 0,234^{* * *}$

Architektur / Bauingenieurwesen $\quad 0,231^{* * *}$

Elektrotechnik / Maschinenbau

Sonstige Ingenieurswissenschaften $0,229^{* * *}$

\begin{tabular}{lccc} 
& & $(0,046)$ \\
\hline Beobachtungen & 3841 & 3841 & 3841 \\
Wald chi2 & $46,25^{* * *}$ & $400,58^{* * *}$ & $724,84^{* * *}$ \\
\hline
\end{tabular}

Robuste Standardfehler in Klammern; Modell 3 enthält 25 Hochschuldummies (Effekte nicht dargestellt); ${ }^{*} p<0,05 ;{ }^{* *} p<0,01 ;{ }^{* * *} p<0,001$

\subsection{Berufseinstieg im Ausland}

Auch bei der Analyse der Determinanten einer ersten Erwerbstätigkeit im Ausland zeigt sich ein höchst signifikanter Effekt der sozialen Herkunft (Tabelle 4). Dieser schwächt sich erwartungsgemäß ab, wenn auf vorherige Mobilitätserfahrungen kontrolliert wird, bleibt aber hoch signifikant. Vorherige Mobilitätserfahrungen haben hier den theoretisch erwarteten Effekt: Absolventen, die einen studienbezogenen Auslandsaufenthalt absolviert oder eine Hochschulzugangsberechtigung außerhalb Bayerns erworben haben, gehen signifikant häufiger ins Ausland als die jeweilige Referenzgruppe.

Weiterhin zeigen sich die vom mikroökonomischen Humankapitalmodell prognostizierten Effekte: Jüngere Absolventen, Singles, Ausländer und Absolventen mit sehr guten Fremdsprachenkenntnissen weisen eine erhöhte Wahrscheinlichkeit auf, ins Ausland zu gehen.

Die international mobilsten Absolventen finden sich bei den Studiengangsgruppen der Architekten und Bauingenieure sowie der Mathematik und den Naturwissenschaften.

Der Effekt der sozialen Herkunft bleibt auch dann signifikant, wenn auf die persönliche Situation, das studierte Fach und den hochschulspezifischen Kontext kontrolliert wird. Dies zeigt, dass die soziale Herkunft sowohl einen signifikanten direkten als auch bedeutsame indirekte - über vorherige Mobilitätserfahrungen vermittelte - Effekte auf die Wahrscheinlichkeit aufweist, eine erste Erwerbstätigkeit im Ausland aufzunehmen. Der Effekt eines homogen 
hohen Status verringert sich hier um ca. 38\%, wenn (in Modell 2) vorherige Mobilitätserfahrungen bei der Analyse berücksichtigt werden.

Tabelle 4: Determinanten des Berufseinstiegs im Ausland, logistische Regression, averaged marginal effects

(Ref. Status homogen niedrig)

Status homogen hoch

Status heterogen

studienbezogener Auslandsaufenthalt

(Ref. HZB in Bayern)

HZB in Restdeutschland

HZB im Ausland

Single

Frau
$(0,009)$

$0,042^{* * *}$

$(0,009)$

$0,020^{*}$

$(0,009)$
Modell 2

$0,026^{* *}$

$(0,009)$

0,013

$(0,009)$

$\begin{array}{cc}0,060^{* * *} & 0,049^{* * *} \\ (0,007) & (0,008)\end{array}$

$0,024^{* *}$

$0,024^{* *}$

$(0,007)$

$(0,008)$

$0,070^{* * *}$

$(0,016)$

0,042

$(0,022)$

$0,020^{* *}$

$(0,007)$

$-0,006$

$(0,007)$

$-0,005^{* *}$

$(0,002)$

(Ref. schlechte Fremdsprachenk.)

sehr gute Fremdsprachenkompetenz

$0,039^{* *}$

$(0,012)$

0,006

$(0,011)$

$0,043^{*}$

$(0,019)$

(Ref. Informatik)

Kunst und Innenarchitektur

$0,060^{*}$

$(0,024)$

0,048

$(0,025)$

Sprach- und Kulturwissenschaften 
Psychologie / Pädagogik / Soziologie / Po-

litikwissenschaften

Soziale Arbeit

Wirtschaftswissenschaften

Mathematik und Naturwissenschaften

Architektur / Bauingenieurwesen

Elektrotechnik / Maschinenbau

\begin{tabular}{lccc}
\hline Beobachtungen & & $(0,022)$ \\
Wald chi2 & 4991 & 4991 & 4991 \\
\hline
\end{tabular}

Robuste Standardfehler in Klammern; Modell 3 enthält 25 Hochschuldummies (Effekte nicht dargestellt); ${ }^{*} p<0,05 ;{ }^{* *} p<0,01 ;{ }^{* * *} p<0,001$

\section{Zusammenfassung}

Der zentrale Fokus dieser Arbeit liegt in der theoretischen und empirischen Durchleuchtung des Einflusses der sozialen Herkunft auf internationale Mobilität während des Studiums und beim Berufseinstieg. Die signifikant positiven Koeffizienten der sozialen Herkunft in den empirischen Analysen zeigen, dass Studierende sowie Hochschulabsolventen mit hoher sozialer Herkunft erstens eher während des Studiums internationale Erfahrungen sammeln zweitens einen größeren Stellensuchradius aufweisen und drittens eher ein Jobangebot im Ausland annehmen. Diese Zusammenhänge erweisen sich als robust, wenn auf vorherige Mobilitätserfahrungen, individuelle Unterschiede, studienfachspezifische Differenzen sowie hochschulspezifische Unterschiede kontrolliert wird. Außerdem unterstreichen die empirischen Ergebnisse, dass die Wahrscheinlichkeit internationaler Mobilität sowie der Stellensuchradius bei Studierenden und Hochschulabsolventen mit vorherigen Mobilitätserfahrungen tendenziell größer ist. Da Studierende und Hochschulabsolventen mit hoher sozialer Herkunft eher über frühere Mobilitätserfahrungen verfügen, bestehen hier also auch indirekte Effekte der sozialen Herkunft. Diese indirekten Effekte sind für Auslandsaufenthalte während des Studiums relativ unbedeutend, gewinnen jedoch an Relevanz, wenn der Stellensuchradius und die Wahrscheinlichkeit eines Berufseinstiegs im Ausland betrachtet werden. 
Diese empirischen Ergebnisse stehen im Einklang mit theoretischen Vorhersagen des mikroökonomischen Humankapitalmodells und bisherigen Forschungsergebnissen zu Migrationsverhalten im Allgemeinen und ergänzen Studien zum Mobilitätsverhalten von Studierenden und Hochschulabsolventen im Besonderen. Im nächsten Abschnitt werden nun Implikationen der Ergebnisse im Hinblick auf die Konsequenzen für soziale Ungleichheit und Anforderungen an weitere Forschung diskutiert.

\section{Diskussion}

Eltern von Studierenden und Hochschulabsolventen mit günstigem sozioökonomischem Hintergrund verfügen über mehr Wissen über die universitäre Umgebung und deren Möglichkeiten ins Ausland zu gehen, eine höhere Wahrscheinlichkeit internationale Erfahrungen gesammelt zu haben und bessere finanzielle Ressourcen. Diese Charakteristiken korrelieren mit Unterstützung durch Informationen, moralischer Unterstützung und finanzieller Unterstützung. Außerdem haben Studierende und Hochschulabsolventen mit günstigen sozioökonomischen Hintergrundcharakteristiken selbst eher bereits Erfahrungen mit internationaler Mobilität gesammelt und verfügen über tendenziell bessere Fremdsprachenkenntnisse (vgl. Lörz / Krawietz 2011). Weitere Forschung muss an dieser Stelle ansetzen, um die relative Bedeutung dieser einzelnen Komponenten für internationale Mobilität im Studium und beim Berufseinstieg sowie für den Stellensuchradius zu klären. ${ }^{15}$

Die Verbindung des mikroökonischen Humankapitalmodells mit der Suchtheorie impliziert außerdem, dass ungleiche Chancen internationaler Mobilität im Studium und beim Berufseinstieg zu differierenden Informationskosten und Mobilitätskosten führen. Daraus resultieren wiederum unterschiedliche Suchradien, Suchdauern und Reservationslöhne. Demzufolge sind für Studierende und Hochschulabsolventen mit stärker ausgeprägten Mobilitätsneigungen langfristig höhere Einkommen zu erwarten (vgl. hierzu DaVanzo 1976, 1983; Faggian et al. 2006; Faggian et al. 2007 b; Newbold 1997). Der Nachweis solcher Einkommensvorteile ist mit erheblichen methodischen Schwierigkeiten verbunden, da international mobile Studierende und Hochschulabsolventen schwer erreicht werden können, eine hoch selektive Gruppe darstellen (vgl. Lörz / Krawietz 2011), eher eine Promotion beginnen (vgl. Messer / Wolter 2007) und zudem international unterschiedliche Preisniveaus berücksichtigt werden müssen. Weiterführende Forschung sollte an dieser Stelle ansetzen, um den Einfluss von Mobilitätserfahrungen im Allgemeinen und internationalen Mobilitätserfahrungen im Besonderen auf die langfristigen Berufschancen von Hochqualifizierten zu untersuchen.

\section{Literatur}

Abraham, Martin / Natascha Nisic (2007): Regionale Bindung, räumliche Mobilität und Arbeitsmarkt Analysen für die Schweiz und Deutschland, in: Swiss Journal of Sociology 33 / 1, S. 69-87.

Abraham, Martin / Katrin Auspurg / Thomas Hinz (2010): Migration Decisions Within Dual-Earner Partnerships: A Test of Bargaining Theory. In: Journal of Marriage and the Family 72 / 4, S. 876-892.

Angrist, Joshua D. / Jorn-Steffen Pischke (2009): Mostly Harmless Econometrics: An Empiricist's Companion. Princeton / NJ.

Auspurg, Katrin / Martin Abraham (2007): Die Umzugsentscheidung von Paaren als Verhandlungsproblem. Eine quasiexperimentelle Überprüfung des Bargaining-Modells, in: Kölner Zeitschrift für Soziologie und Sozialpsychologie 59, S. 271-293.

Bailey, Adrian (1993): Migration history, migration behavior, and selectivity, in: The Annals of Regional Science 27, S. 315-326.

15 Ein erster Schritt in diese Richtung wurde durch die Studie von Lörz / Krawietz (2011) unternommen. 
Best, Henning / Christof Wolf (2010): Logistische Regression, in: Dies. (Hrsg.), Handbuch der sozialwissenschaftlichen Datenanalyse, Wiesbaden, S. 827-854.

Becker, Rolf (2000): Klassenlage und Bildungsentscheidungen. Eine empirische Anwendung der WertErwartungstheorie, in: Kölner Zeitschrift für Soziologie und Sozialpsychologie 52, S. 450-474.

Behnke, Joachim (2005): Lassen sich Signifikanztests auf Vollerhebungen anwenden? Einige Anmerkungen, in: Politische Vierteljahresschrift 46, S.O1-O15.

Birg, Herwig / Jürgen Flöthmann (1992): Biographische Determinanten der räumlichen Mobilität, in: Akademie für Raumforschung und Landesplanung (Hrsg.), Regionale und biographische Mobilität im Lebensverlauf, Hannover, S. 6-26.

Blien, Uwe / Michael Wiedenbeck (2002): Mehrebenenanalyse, in: Gerhard Kleinhenz (Hrsg.), IABKompendium Arbeitsmarkt- und Berufsforschung, BeitrAB 250, S. 309-324.

Blossfeld, Hans-Peter (1993): Die berufliche Erstausbildung Jugendlicher im internationalen Vergleich, in: Peter Diepold / Adolf Kell (Hrsg.), Entwicklungen in der Berufsausbildung. Deutsche Berufsausbildung zwischen Modernisierung und Modernitätskrise im Kontext der Europäischen Integration. Beiheft 11 zur Zeitschrift für Berufs- und Wirtschaftspädagogik, Stuttgart, S. 23-40.

Bourdieu, Pierre. (1982). Der Habitus und der Raum der Lebensstile, in: Pierre Bourdieu, Die feinen Unterschiede, Frankfurt / Main, S. 277-322.

Broscheid, Andreas / Thomas Gschwend (2005): Zur statistischen Analyse von Vollerhebungen, in: Politische Vierteljahresschrift 46, S.O16-O26.

Brüderl, Josef (2010): Kausalanalyse mit Paneldaten, in: Henning Best / Christof, Wolf(Hrsg.), Handbuch der sozialwissenschaftlichen Datenanalyse, Wiesbaden, S. 963-994.

Chiswick, Barry R (1991): Speaking, Reading, and Earnings among Low-Skilled Immigrants, in: Journal of Labour Economics 9, S. 149-170.

Curran, Sara R. / Estela Rivera-Fuentes (2003): Engendering Migrant Networks: The case of Mexican Migration, in: Demography 40 / 2, S. 289-307.

DaVanzo, Julie (1976): Differences between Return and Non-Return Migration: An Econometric Analysis, in: International Migration Review 10, S. 13-27.

DaVanzo, Julie (1978): Does employment affect migration? Evidence from microdata, in: Review of Economics and Statistics 60, S. 504-514.

DaVanzo, Julie (1983): Repeat Migration in the United States: Who Moves Back and Who Moves On?, in: Review of Economics and Statistics 65, S. 552-559.

Del Rey Poveda, Alberto (2007): Determinants and Consequences of Internal and International Migration: The Case of rural Populations in the South of Veracruz, Mexico, in: Demographic Research 16, S. 287-314.

Diehl, Claudia / David Dixon (2005): Zieht es die Besten fort? Ausmaß und Formen der Abwanderung deutscher Hochqualifizierter in die USA, in: Kölner Zeitschrift für Soziologie und Sozialpsychologie 57, S. 714-734.

Enders, Jürgen / Lutz Bornmann (2002): Internationale Mobilität bundesdeutscher Promovierter - Eine Sekundäranalyse der Kaseler Promoviertenstudie, in: Mitteilungen aus der Arbeitsmarkt und Berufsforschung 35/1, S. 60-73.

Fabian, Gregor / Karl-Heinz Minks (2008): Muss i denn zum Städtele hinaus? Erwerbsmobilität von Hochschulabsolventen, in: HIS Magazin 3, S. 4-5.

Faggian, Alessandra, Philip McCann und Stephen C. Sheppard (2007 a): Some Evidence that Women are More Mobile than Men: Gender Differences in UK Graduate Migration Behaviour, in: Journal of Regional Science 47, S.517-539.

Faggian, Alessandra / Philip McCann / Stephen C. Sheppard (2007 b): Human Capital, Higher Education and Graduate Migration: An Analysis of Scottish and Welsh Students, in: Urban Studies 44, S. 2511-2528. 
Faggian, Alessandra / Philip McCann (2009): Human Capital, graduate migration and innovation in British regions, in: Cambridge Journal of Economics 33, S. 317-333.

Falk, Susanne / Fabian Kratz (2009): Regionale Mobilität von Hochschulabsolventen beim Berufseinstieg, in: Beiträge zur Hochschulforschung 3, S. 52-67.

Hartmann, Michael (2002): Der Mythos von den Leistungseliten. Frankfurt / Main.

Hartmann, Michael (2010): Achievement or Origin: Social Background and Ascent to Top Management, in: Talent Development \& Excellence 2, S. 105-117.

Hartmann, Michael / Johannes Kopp (2001): Elitenselektion durch Bildung oder durch Herkunft? Promotion, soziale Herkunft und der Zugang zu Führungspositionen in der deutschen Wirtschaft, in: Kölner Zeitschrift für Soziologie und Sozialpsychologie 53, S. 436-466.

Henz, Ursula / Ineke Maas (1995): Chancengleichheit durch die Bildungsexpansion?, in: Kölner Zeitschrift für Soziologie und Sozialpsychologie 47, S. 605-633.

Herzog, Henry W. / Richard A. Hofler / Alan M. Schlottman (1985): Life on the Frontier: Migrant Information, Earnings and Past Mobility, in: Review of Economics and Statistics 67, S. 373-82.

Hillmert, Steffen / Marita Jacob (2005): Institutionelle Strukturierung und inter-individuelle Variation. Zur Entwicklung herkunftsbezogener Ungleichheiten im Bildungsverlauf, in: Kölner Zeitschrift für Soziologie und Sozialpsychologie 57, S. 414-442.

Isserstedt, Wolfgang / Judith Link (2008): Internationalisierung des Studiums - Ausländische Studierende in Deutschland - Deutsche Studierende im Ausland, abrufbar unter: http://www.Studierendewerke.de/ pdf/Sonderbericht_Internationalisierung.pdf, letztes Abrufdatum: 6.12.2011.

Jahr, Volker / Harald Schomburg / Ulrich Teichler (2002): Mobilität von Hochschulabsolventinnen und -absolventen in Europa, in: Lutz Bellmann / Johannes Velling (Hrsg.), Beiträge zur Arbeitsmarkt- und Berufsforschung: Arbeitsmärkte für Hochqualifizierte, Nürnberg: Bundesanstalt für Arbeit, S. 317-347

Köhler, Helmut (1992): Bildungsbeteiligung und Sozialstruktur in der Bundesrepublik. Zu Stabilität und Wandel der Ungleichheit von Bildungschancen, Berlin: Max-Planck-Institut für Bildungsforschung.

Konietzka, Dirk (1999): Ausbildung und Beruf. Die Geburtsjahrgänge 1919-1961 auf dem Weg von der Schule in das Erwerbsleben, Opladen.

Krais, Beate (1996): Bildungsexpansion und soziale Ungleichheit in der Bundesrepublik Deutschland, in: Axel Bolder / Helmut Heid / Walter R. Heinz / Günter Kutscha / Helga Krüger / Artur Meier / Klaus Rodax (Hrsg.), Die Wiederentdeckung der Ungleichheit. Aktuelle Tendenzen in Bildung für Arbeit. Jahrbuch Bildung und Arbeit, Opladen, 118-146.

Kühnel, Steffen M. / Dagmar Krebs (2010): Multinomiale und ordinale Regression, in: Henning Best, Henning / Christof Wolf (Hrsg.), Handbuch der sozialwissenschaftlichen Datenanalyse, Wiesbaden, S. 855-886.

Lörz, Markus (2008): Räumliche Mobilität beim Übergang ins Studium und im Studienverlauf, in: Bildung und Erziehung 61 / 4, S. 413-436.

Lörz, Markus / Marian Krawietz (2011): Internationale Mobilität und soziale Selektivität: Ausmaß, Mechanismen und Entwicklung herkunftsspezifischer Unterschiede zwischen 1990 und 2005.

Long, Scott J. / Jeremy Freese (2006): Regression Models for Categorical Dependent Variables Using Stata, 2. Aufl., Texas.

Massey, Douglas S. / Felipe García España (1987): The Social Process of International Migration, in: Science 237, S. 733-738.

Massey, Douglas S. / Kristin E. Espinosa (1997): What's Driving Mexico-US Migration? A Theoretical, Empirical, and Policy Analysis, in: American Journal of Sociology 102, S. 939-999.

Mayer, Karl-Ulrich (1991): Lebensverlauf und Bildung. Ergebnisse aus dem Forschungsprojekt „Lebensverläufe und gesellschaftlicher Wandel“" des Max-Planck-Instituts für Bildungsforschung, in: Unterrichtswissenschaft 19, S. 313-332. 
Mayer, Karl Ulrich / Hans-Peter Blossfeld, (1990): Die gesellschaftliche Konstruktion sozialer Ungleichheit im Lebensverlauf, in: Peter A. Berger / Stefan Hradil (Hrsg.), Lebenslagen, Lebensläufe, Lebensstile. Sonderband 7 der Sozialen Welt, Göttingen, S. 297-318.

Menard, Scott (2002): Applied Logistic Regression Analysis, in: Michael S. Lewis-Beck (Hrsg.), Series: Quantitative Applications in the Social Sciences, Thousand Oaks.

Messer, Dolores / Stefan C. Wolter (2007): Are student exchange programs worth it?, in: Higher Education 54, S. 647-663.

Meulemann, Heiner (1995): Die Geschichte einer Jugend. Lebenserfolg und Erfolgsdeutung ehemaliger Gymnasiasten zwischen dem 15. und 30. Lebensjahr, in: Opladen.

Mincer, Jacob (1978): Family Migration Decisions, in: Journal of Political Economy 86, S. 749-773.

Molho, Ian (1986): Theories of Migration: A Review, in: Scottish Journal of Political Economy 33, S. 396-419.

Mood, Carina (2010): Logistic Regression: Why we cannot do what we think we can do, and what we can do about it, in: European Sociological Review 26 / 1, S. 67-82.

Müller, Walter / Dietmar Haun (1994): Bildungsungleichheit im sozialen Wandel, in: Kölner Zeitschrift für Soziologie und Sozialpsychologie 46, S. 1-42.

Newbold, Bruce K. (1997): Primary, return and onward migration in the US and Canada: is there a difference?, in: Papers in Regional Science 76, S. 175-198.

Ohnmacht, Timo / Andreas Frei / Kay W. Axhausen (2008): Mobilitätsbiografie und Netzwerkgeografie: Wessen soziale Beziehungen sind räumlich dispers?, in: Swiss Journal of Sociology 34, S. 131-164.

Pineda, Pilar, Victoria Moreno und Esther Belvis (2008): The Mobility of University Students in Europe and Spain, in: European educational research journal 7 / 3, S. 273-288.

Rabe-Hesketh, Sophia / Anders Skrondal (2008): Multilevel and Longitudinal Modelling Using STATA, Texas.

Rindskopf, David (2004): Trends in Categorical Data Analysis: New, Semi-New, and Recycled Ideas, in: David Kaplan (Hrsg.), The Sage Handbook of Quantitative Methodology for the Social Sciences, Thousand Oaks, S. 137-150.

Rodax, Klaus (1995): Soziale Ungleichheit und Mobilität durch Bildung in der Bundesrepublik Deutschland, in: Österreichische Zeitschrift für Soziologie 20, S. 3-27.

Rolfes, Manfred (1996): Regionale Mobilität und akademischer Arbeitsmarkt. Osnabrücker Studien zur Geographie, Osnabrück.

Savage, Mike (1988): The missing link? The relationship between spatial mobility and social mobility, in: The British Journal of Sociology XXXIX, S. 554-577.

Schimpl-Neimanns, Bernhard (2000): Soziale Herkunft und Bildungsbeteiligung. Empirische Analysen zu herkunftsspezifischen Bildungsungleichheiten zwischen 1950 und 1989, in: Kölner Zeitschrift für Soziologie und Sozialpsychologie 52, S. 636-669.

Shavit, Yossi / Hans-Peter Blossfeld (1993): Persistent inequalities: Changing educational stratification in thirteen countries, Boulder / Col..

Simpson, Wayne (1992): Urban Structure and the Labor Market: Worker Mobility, Commuting, and Underemployment in Cities, Oxford.

Sjaastad, Larry A. (1962): The Costs and Returns of Human Migration, in: The Journal of Political Economy 70, S. 80-93.

Snijders, Tom / Roel Bosker (1999): Multilevel Analysis: An Introduction to Basic and Advanced Multilevel Modeling, London.

Speare, Alden (1971): A Cost-Genefit Model of Rural to Urban Migration in Taiwan, in: Population Studies 25, S. 117-130. 
Speare, Alden (1974): Residential Satisfaction as an Intervening Variable in Residential Mobility, in: Demography 11, S. 173-188.

Tutz, Gerhard (2000): Die Analyse kategorialer Daten, München.

Wagner, Michael (1989): Räumliche Mobilität im Lebenslauf, Stuttgart.

Wooldridge, Jeffrey M. (2002): Econometric Analysis of Cross Section and Panel Data, Cambridge / MA.

Wooldridge, Jeffrey M. (2003): Introductory Econometrics. A Modern Approach, Mason.

Fabian Kratz Ludwig-Maximilians-Universität München Institut für Soziologie Konradstr. 6 80801 München fabian.kratz@soziologie.uni-muenchen.de 